\title{
Enteral Administration of Agar as an Effective Adjunct to Phototherapy of Neonatal Hyperbilirubinemia
}

\author{
GERARD B. ODELL, ${ }^{(34)}$ GARY R. GUTCHER, PETER F. WHITINGTON, AND GLORIA YANG \\ University of Wisconsin School of Medicine, Department of Pediatrics, 600 Higland Avenue, \\ Madison, Wisconsin USA
}

\begin{abstract}
Summary
Phototherapy increases the biliary excretion of unconjugated bilirubin. In this form, bilirubin would be subject to enterohepatic circulation, and the true efficacy of phototherapy would be blunted. We tested the hypothesis that sequestration of lumenal unconjugated bilirubin by enteral agar administration would enhance the efficacy of phototherapy in jaundiced infants. Fifty-two infants were studied, 21 control and 31 agar-supplemented. The birth weights, sex distribution, and postnatal age at onset of phototherapy did not differ between the two groups of infants; pre- and postphototherapy bilirubin concentrations also did not differ between the groups. The bile acid concentrations and bilirubin saturation indices were also similar. The rate of declination of the plasma bilirubin concentrations after $24 \mathrm{~h}$ of phototherapy was greater and significantly more uniform in the agar-supplemented infants $(-1.59 \pm 2.3$ versus $-2.51 \pm 1.44)$. Stool frequencies were greater in control infants (5.5 versus 4.3 per $24 \mathrm{~h}$ ) whereas fecal bilirubin excretions were greater in agar-supplemented infants during the second day of phototherapy $\left(1.32\right.$ versus $\left.3.29 \mathrm{mg} \cdot \mathrm{kg}^{-1} \cdot 24 \mathrm{~h}^{-1}\right)$. Agar supplementation reduced the duration of phototherapy by $23 \%$ (37.6 \pm 3.2 versus $48.1 \pm 5.0 \mathrm{~h})$.
\end{abstract}

Irradiance from broad-spectrum fluorescent lights (phototherapy) is a universally applied therapy for the treatment of neonatal hyperbilirubinemia $(1,2)$. Since its original description in 1958 (3), there has been considerable inquiry as to the mechanism(s) by which phototherapy is able to effect a reduction of visible icterus and serum bilirubin concentration in jaundiced infants. Ostrow (4) initially demonstrated the association of an increased biliary excretion of unconjugated bilirubin with fluorescent irradiation of the jaundiced Gunn rat. Lund and colleagues (5) subsequently made qualitatively similar observations in human infants. A plausible mechanism to explain how irradiation of the jaundiced infant's dermis results in hepatic excretion of unconjugated bilirubin has received experimental validation $(6,7)$.

It is thought that bilirubin IX $\alpha$ in its natural Z-Z configuration undergoes a photon-induced $\mathrm{Z} \rightarrow \mathrm{E}$ geometric isomerization to more water-soluble products, "photobilirubins" $(8,9)$. These photobilirubins presumably dissociate from their binding sites in skin and diffuse into the circulation. Their subsequent clearance by the liver into the bile can be accomplished without further biotransformation as an organic anion comparable to indocyanine green and dibromosulfophthalein (10).

The photobilirubins are unstable and revert to the natural isomer in either the bile or intestinal lumen. Such a reversion would render the excreted bilirubin susceptible to enterohepatic circulation (11), and the true efficacy of phototherapy on the reduction of serum bilirubin concentration would be blunted. But if the lumenal unconjugated bilirubin could be sequestered from the enterohepatic circulation, the effectiveness of phototherapy might be increased.

The sequestration of nonconjugated bilirubin from the entero- hepatic circulation was initially demonstrated by the oral administration of agar to term infants (12). Subsequent reports have been contradictory. In one, the agar was added directly to the infants' formulas, which makes it doubtful that it was actually ingested by the infants; powdered agar does not readily dissolve and would be unlikely to pass the nipple of the formula bottle (13). In two reports, enteral agar was associated with a statistically significant lowering of serum bilirubin concentrations during neonatal life, but its effectiveness was not acclaimed $(14,15)$. In three other reports, the serum bilirubin concentrations of the agartreated infants did not differ from those of the controls (16-18). In none of the conflicting studies cited was the agar pretested for its binding affinity for bilirubin, and bile pigment excretions in the stools were not measured. This laboratory has reported that agars preselected for their high binding affinity for bilirubin by in vitro testing (19) effectively reduce serum bilirubin concentrations in experimental animals and the Crigler-Najjar syndrome $(20,21)$ The lowering of serum bilirubin concentrations was also associated with greater stool excretions of bile pigment.

We currently report that enteral administration of agar to infants with neonatal hyperbilirubinemia significantly curtailed the number of hours of phototherapy necessary to reduce their serum bilirubin concentrations to levels that are conventionally considered nontoxic.

\section{PATIENTS AND METHODS}

Study patients were neonatal infants whose concentrations of serum bilirubin reached levels that caused their attending pediatricians to order the use of phototherapy. In total, the patients of eight staff pediatricians were involved: four full-time physicians of the neonatal service and four nursery-privileged university clinic or privately practicing pediatricians. The decisions of these eight pediatricians as to when to initiate or terminate phototherapy was based on the infants' serum concentrations of bilirubin determined in the hospitals' clinical laboratory and are unrelated to the protocol of this study. It is common practice in the nursery to initiate continuous phototherapy in term infants when serum bilirubin levels exceed $14 \mathrm{mg} / \mathrm{dl}$ and with preterm babies when the concentration is above $10 \mathrm{mg} / \mathrm{dl}$. Phototherapy is discontinued in both groups when the level returns to 11 and $10 \mathrm{mg} / \mathrm{dl}$, respectively.

At the time of initiation of phototherapy, at $24 \mathrm{~h}$ during its usage and at its termination, an additional $0.4 \mathrm{ml}$ of capillary blood was collected into Natelson capillary tubes for the purposes of this investigation and refrigerated in the dark. Whenever possible, these collections were concurrent with routine blood samplings every 8 hours, required to monitor the infant's bilirubin concentrations in the clinical laboratory. These three samples were analyzed separately in the authors' research labortory. During phototherapy, all stools were collected by inclusion of a thin plastic liner in the diaper. The times of stool passage were recorded for each infant, and the samples were folded within the liner, placed in a light-resistant bag and stored in a light-protected 
polystyrene bucket. All blood and stool samples were collected daily for analysis in the research laboratory.

A total of 67 infants were prospectively enrolled in the study: 32 control and 35 agar-supplemented babies. For a subject to be included in the study, enteral feedings (either by nipple or by nasogastric tube) had to be in use, and no demonstrable evidence of excessive bilirubin formation was present. None of the infants had obvious hematomas, anemia or isoimmune blood group incompatibilities. All had negative Coombs tests of their cord blood. Blood cultures, when performed, were sterile. Retrospective analysis according to the above criteria required elimination of 11 infants from the controls and four from the agar study groups.

The study protocol had prior approval of the Hospital and University Human Subjects Committees, and the parents gave their informed consent for inclusion of their infants in the study. The assignment of an infant to control or agar-supplemented groups was based on the last digit of the infant's history number: if even, the infant was placed in the control group; if odd, the infant received agar.

One of the authors (GRG) or his designee obtained parental consent, without revealing to the parents or the attending physician whether their infant was to be in the control or agar-supplemented group. The protocol could not be double-blinded, because we could not devise a satisfactory placebo for control infants; consequently, the nursery personnel, parents and attending physicians became aware of the differences once the protocol was begun. All infants were appropriate for gestational age: 13 were pre-term in the agar-tested group, and eight were pre-term in the control group.

A USP agar (Amend Drug and Chemical Co.) was employed. By in vitro analysis (19), it demonstrated a binding affinity for bilirubin of $\mathrm{Ka}=10^{5}$, with 25.5 moles of independent binding sites per $50,000 \mathrm{~g}$ of agar, as extrapolated from the Scatchard plot of the adsorption isotherm. The calcium content was 4.2 moles per $50,000 \mathrm{~g}$ of agar, which was determined by atomic absorption spectroscopy on an acid digest of the agar (19). The agar (250 mg) was preweighed and transferred to individual No. 0 gelatin capsules (Eli Lilly and $\mathrm{Co}$.). Those infants in the agar supplementation group received $250 \mathrm{mg}$ every $8 \mathrm{~h}$ during phototherapy. The gelatin capsule of agar was emptied into $5 \mathrm{ml}$ of the enteral feeding mixture of the infant and suspended by vigorous stirring. The suspension was administered by medicine dropper to nipple-fed infants or through the nasogastric tube-fed infants and immediately followed by completion of the feeding.

The phototherapy unit consisted of fluorescent cool-white (GE F20T12-CW) and blue lamps (Westinghouse F20T12/B). It provided a skin-level irradiance of $1.5 \mathrm{~mW} / \mathrm{cm}^{2}$ in the $400-500 \mathrm{~nm}$ band pass and was periodically checked by a previously described light meter (22).

During phototherapy, the infant's eyes were protected from the light source by loosely-fitted opaque eye patches, and the gonads were shielded by normal diapering.

Chemical analysis of sera. The capillary blood samples were centrifuged at $3000 \times g$ for $10 \mathrm{~min}$. The direct $(5 \mathrm{~min})$ and total bilirubin concentrations were determined on the supernatant plasma $(40 \mu \mathrm{L})$ by a micro-modification of the Malloy and Evelyn technique $(21,23)$. The relative saturation of the serum albumin with bilirubin was determined by the Salicylate Saturation Index, $40 \mu \mathrm{L}$ of plasma (21). Total serum bile acid concentrtion $(0.1 \mathrm{ml}$ of plasma when available) was measured by the fluorometric assay of Schwartz et al. (24), employing $3 \alpha$-steroid-dehydrogenase, which was kindly provided by Dr. Paul Talalay (25).

The stool samples were pooled into 24-h lots. Their weights were determined from the weights of the plastic liners before and after transfer of the stool sample to homogenizer vessels. On 50\% ethanol homogenates of the pooled sample, the bilirubin content was measured colorimetrically, as previously validated (12). Completeness of stool collections was verified by identifying one sample with each of the recorded stool passage entries on the infant's protocol sheet.

Statistical comparisons were made by use of Student's $t$ test for unpaired values between the two infant populations and paired $t$ test when comparisons were performed on the same infant before and after phototherapy. Chi-square analyses incorporating a Yates correction was also applied when pertinent, as was the variance ratio F-test; $\boldsymbol{P}$ values less than 0.05 were considered significant $(26,27)$.

\section{RESULTS}

Fifteen of the 67 infants prospectively enrolled in the study had to be excluded after their medical records were reviewed retrospectively. Isoimmune hemolytic disease with a positive cord blood Coombs test was found in four infants. Postphototherapy bilirubin samples had not been collected in three. The agar had not been given in two. Respiratory distress syndrome complicated neonatal courses in three infants. In one infant, the serum bilirubin concentration was less than $9.0 \mathrm{mg} / \mathrm{dl}$ at the start of phototherapy. Another infant was enrolled in a concurrent perinatal study in which the mother received steroid therapy before delivery. Last, one infant developed a skin rash and was removed from phototherapy before the bilirubin level had shown significant reduction.

The comparability of the 52 remaining control and agar-supplemented infants is summarized in Table 1. As indicated in the far righthand column, there were no statistically significant differences in the measured characteristics of the two infant populations with respect to weights, sex distribution, age, or bilirubin concentrations at the beginning and completion of phototherapy.

Table 2 records the additional plasma determinations performed on the infant populations. The relative saturations of plasma albumin with bilirubin were not significantly different between the infant groups; however, the decrease in saturation index of the agar-supplemented infants was significant $(6.2$ to $3.8, P<0.01)$ whereas that in the controls was not $(P>0.05)$. Likewise, the decrement in the plasma bilirubin concentrations $(-1.589$ versus -2.508 ) after $24 \mathrm{~h}$ of phototherapy was far more significant in the

Table 1. Comparative measurements in the control and agar-supplemented infants whose neonatal hyperbilirubinemia was treated by phototherapy

\begin{tabular}{|c|c|c|c|}
\hline & Control $(n)^{1}$ & $\begin{array}{c}\text { Agar- } \\
\text { supplemented }(n)\end{array}$ & Significance \\
\hline Birth weight $(\mathrm{kg})$ & $2.85 \pm 0.66^{3}(21)$ & $2.71 \pm 0.91(31)$ & $\mathrm{NS}^{2}$ \\
\hline Males & 13 & 18 & NS \\
\hline \multicolumn{4}{|c|}{ At start of phototherapy } \\
\hline Age (days) & $3.37 \pm 1.51(21)$ & $3.36 \pm 0.96(31)$ & NS \\
\hline Bilirubin (mg/dl) & $13.6 \pm 3.13(21)$ & $13.7 \pm 2.50(31)$ & NS \\
\hline
\end{tabular}

\footnotetext{
${ }^{1}(n)$, number of patients.

${ }^{2} \mathrm{NS}$, not significant.

${ }^{3}$ Standard deviation.
} 
agar-treated group $(P=0.05$ versus $P<0.001)$, and the variance ratio $F$-test indicated the decrease in serum bilirubins was significantly more uniform in the agar-supplemented infants. The discrepancy between Tables 1 and 2 in the number of infants, on whom such bilirubin comparisons could be made, occurred because not all the infants required $24 \mathrm{~h}$ of phototherapy.

None of the infants exhibited abnormal elevations in their plasma bile acid concentrations (normal $=20 \mu \mathrm{M}$ during neonatal life by the $3 \alpha$-steroid dehydrogenase assay), and none had "directreacting" bilirubin concentrations that exceeded $1.0 \mathrm{mg} / \mathrm{dl}$ during or after the course of phototherapy. The latter two measurements indicated that laboratory evidence of cholestasis did not occur in association with the phototherapy.

Table 3 records the excretory measurements made on the infants during phototherapy. As indicated, stool frequency was significantly greater during the 2 days of phototherapy in the control infants, but total weights for the 2 days did not differ. Of importance was the observations that the agar-treated infants had significantly greater bilirubin excretions during the second day of phototherapy, and the differences in pigment excretion between days \#1 and \#2 were greater in the agar-treated infants. Some caution is warranted in the pigment excretion data because the number of infants, on whom satisfactory 48-h collections were made, is small. Three of the control infants had significant diarrhea during phototherapy that did not permit satisfactory collections. Although diarrhea did not occur in the agar-treated infants, phototherapy had been stopped before $36 \mathrm{~h}$ and, consequently, so were the stool collections.

Tables 4 and 5 illustrate the influence of agar supplementation on the duration of phototherapy. Table 4 demonstrates that the mean duration of phototherapy was significantly less $(37.6 \mathrm{~h}$ versus $48.1 \mathrm{~h}$ ) when agar supplementation was employed, despite the large standard deviations in both groups. When the duration of phototherapy was analyzed separately for term and preterm infants, the difference by $t$ test was significant $(P<0.05)$ in term infants $(>2.5 \mathrm{~kg})$ but did not reach significance in the preterm group $(<2.5 \mathrm{~kg}$ ); however, the number involved were small in the latter group of infants.

Table 5 summarizes the influence of agar on duration of phototherapy in the preterm and term infant populations. As illustrated, the agar supplementation shows similar trends in both population groups, but because each of the $2 \times 2$ contingency tables contains cells with numbers less than 5 , a chi-square analysis could not be properly applied until the populations were combined [Table 5(C)]. The latter portion of the Table demonstrates that $71 \%$ of the control infants required more than $36 \mathrm{~h}$ of phototherapy to reduce their bilirubin concentrations below $11 \mathrm{mg} / \mathrm{dl}$. In contrast, only $39 \%$ of the agar-supplemented infants required more than $36 \mathrm{~h}$ to achieve comparable reductions.

\section{DISCUSSION}

The current observations indicate that enteral supplementation with an agar of high affinity for bilirubin can improve the efficiency of phototherapy in the management of neonatal hyperbilirubinemia. A $23 \%$ reduction $(37.6$ versus $48.1 \mathrm{~h})$ in the duration of phototherapy was realized in the supplemented infants. These mean time values were significantly different by both $t$ test and chi-square analyses. Although the mean serum bilirubin concentrations $24 \mathrm{~h}$ after the initiation of phototherapy were significantly lower in the agar-supplemented group and the rate of decline greater, the mean difference between the control and agar-treated infants did not reach statistical significance. The variance ratio analysis illustrated a significantly more uniform rate of declination

Table 2. Plasma measurements of the infants treated by phototherapy

\begin{tabular}{|c|c|c|c|}
\hline & Control & $\begin{array}{c}\text { Agar- } \\
\text { supplemented }\end{array}$ & Significance \\
\hline \multicolumn{4}{|l|}{ Salicylate saturation index (\%) } \\
\hline At start of phototherapy & $5.8 \pm 2.63(19)$ & $6.2 \pm 2.91(28)$ & NS \\
\hline \multicolumn{4}{|l|}{ Bilirubin (mg/dl) } \\
\hline At start of phototherapy & $13.56 \pm 3.08(19)$ & $13.87 \pm 2.57(25)$ & NS \\
\hline After $24 \mathrm{~h}$ of phototherapy & $11.97 \pm 2.70(19)$ & $11.36 \pm 2.01(25)$ & NS \\
\hline $\begin{array}{l}\text { Decrement in bilirubin concen- } \\
\text { tration after } 24 \mathrm{~h} \text { of photo- } \\
\text { therapy }(\mathrm{mg} / \mathrm{dl})\end{array}$ & \multicolumn{2}{|c|}{ Variance ratio: $F=2.568$} & $P<0.05$ \\
\hline \multicolumn{4}{|l|}{ Bile acids $(\mu \mathrm{M})$} \\
\hline At start of phototherapy & $21.1 \pm 13.20(9)$ & $13.4 \pm 10.11(12)$ & NS \\
\hline At end of phototherapy & $12.8 \pm 4.74(8)$ & $16.0 \pm 10.06(15)$ & NS \\
\hline
\end{tabular}

Table 3. Excretory measurements during phototherapy treatment of control and agar-supplemented infants with neonatal hyperbilirubinemia

\begin{tabular}{|c|c|c|c|}
\hline & Control & Agar-supplemented & Significance \\
\hline \multicolumn{4}{|l|}{$\begin{array}{l}\text { Stool frequency } \\
\text { (no. } / 24 \mathrm{~h})\end{array}$} \\
\hline Day \#1 & $5.5 \pm 2.98(17)$ & $4.5 \pm 2.32(24)$ & NS \\
\hline Mean of days \#1, 2 & $5.52 \pm 3.23(27)$ & $4.35 \pm 2.21(34)$ & $P=0.05$ \\
\hline \multicolumn{4}{|l|}{ Stool weight $(\mathrm{g})$} \\
\hline Day \#1 & $10.07 \pm 9.36(16)$ & $8.33 \pm 5.81(20)$ & NS \\
\hline \multicolumn{4}{|l|}{$\begin{array}{l}\text { Bile pigment excretion } \\
\left(\mathrm{mg} \cdot \mathrm{kg}^{-1} \cdot 24 \mathrm{~h}^{-1}\right)\end{array}$} \\
\hline Day \#1 & $1.90 \pm 1.08(16)$ & $1.41 \pm 0.86(19)$ & NS \\
\hline Day \#2 & $1.32 \pm 0.87(8)$ & $3.29 \pm 2.51(10)$ & $P=0.025$ \\
\hline$\Delta$ Day 2-Day 1 & $-0.36 \pm 0.55(8)$ & $+1.20 \pm 2.16(7)$ & $P<0.05$ \\
\hline
\end{tabular}


Table 4. Influence of agar supplementation on the duration of phototherapy

\begin{tabular}{cccc}
\hline & Control & Agar-supplemented & Significance \\
\hline $\begin{array}{c}\text { Duration of phototherapy } \\
\text { (in hours) }\end{array}$ & $48.06 \pm 22.98(21)$ & $37.56 \pm 17.99(31)$ & $\mathrm{t}=1.85, P<0.05$ \\
\hline
\end{tabular}

Table 5. Chi-square analysis of the influence of agar supplementation on the duration of phototherapy ${ }^{1}$

\begin{tabular}{lrrr}
\hline & $<36 \mathrm{~h}$ & $>36 \mathrm{~h}$ & Total \\
\hline (A) Pre-term infants & & & \\
Control & 2 & 6 & 8 \\
Agar-supplemented & 8 & 5 & 13 \\
$\quad$ Total & 10 & 11 & 21 \\
(B) Term infants & & & \\
Control & 4 & 9 & 13 \\
Agar-supplemented & 11 & 7 & 18 \\
$\quad$ Total & 15 & 16 & 31 \\
(C) Combined population & & & \\
Control & 6 & 15 & 21 \\
Agar-supplemented & 19 & 12 & 31 \\
Total & 25 & 27 & 52 \\
\hline
\end{tabular}

${ }^{1} \chi^{2}=4.14, P<0.05$.

of the serum bilirubin concentrations in the agar-supplemented infants.

The lowering of the serum bilirubin concentrations during phototherapy was associated with simultaneous decreases in the saturation of albumin with bilirubin in both infant populations, as reflected in the lowering of the saturation indices. These findings are consistent with an improved clearance of bilirubin from the circulation, and the reduction in the saturation index was greater $(P<0.01)$ in the agar-treated infants; moreover, the significantly greater excretion of bile pigment during the second $24 \mathrm{~h}$ of treatment $(3.29$ versus $1.32 \mathrm{mg} / \mathrm{kg} / 24 \mathrm{~h}$ ) attests to the enhanced clearance in the agar-supplemented infants. One would not expect a greater stool pigment excretion to be evident in the first day of supplementation, because an 8-12-h transit time is required before the sequestered bilirubin would be evacuated from the colon.

Two previous reports failed to show any significant influence on serum bilirubin concentrations when agar was used in conjunction with phototherapy in either juandiced newborns (18) or a patient with nonhemolytic hyperbilirubinemia (28). In the former study, the agar was not tested for its binding affinity, and in the latter report, only $1 \mathrm{~g} / \mathrm{kg}$ of agar was used, whereas $2 \mathrm{~g} / \mathrm{kg}$ was found necessary to be effective in older infants with the Crigler-Najjar syndrome (20). The pretesting of the agar and its dosage appear to be critical in the use of agar to enhance the clearance of bilirubin.

The proposed mechanism by which enteral agar improves the clearance of bilirubin is based on its high binding affinity for bilirubin and sequestration from enterohepatic circulation of the increased quantities of nonconjugated bilirubin delivered to the intestinal lumen during phototherapy. It is not known whether "photobilirubins" bind to agar. By analogy to other water-soluble organic anions, such as salicylates, phenosulfophthalein and bilirubin glucuronides, which do not bind to agar (19), one might infer that the water-soluble photoisomers of bilirubin would likewise not be sequestered. More experimental evidence is required to justify such an inference. The rapidity at which the photobilirubins revert to the stable isomer in vitro would indicate binding of photobilirubin is not necessary $(8,9,29)$.

Phototherapy has been associated with an increased stool frequency and a reduced intestinal transit time $(30,31)$. This diarrhea has been demonstrated in experimental animal studies to be associated with an increased net secretion of salt and water in response to the intralumenal concentrations of unconjugated bilirubin (32). This "secretory" diarrhea has also been reversed by concurrent perfusion of agar with the lumenal perfusion of bilirubin (33). The current clinical observations are consistent with these animal studies. The agar-treated infants had fewer stools and no diarrhea whereas the control infants had a greater stool frequency, and three had diarrhea.

The present study also included plasma measurements for conjugated bilirubin and bile acids to detect evidence of cholestasis associated with phototherapy. In cholestatic liver disease, elevations of the direct-reacting bilirubin and bile acid concentrations are common findings that reflect impairment of hepatocyte canalicular excretory function. In no instance did we find evidence of cholestasis.

Although the number of infants studied in the present report is relatively small, the results do demonstrate that blocking the enterohepatic circulation of bilirubin is a logical means of reducing the duration of hospitalization of infants whose neonatal hyperbilirubinemia is treated by phototherapy.

\section{REFERENCES AND NOTES}

1. Final report on the Committee on Phototherapy in the Newborn. Division of Medical Sciences, Assembly of Life Sciences, National Research Council. (National Academy of Sciences, Washington D.C., 1974).

2. Brown, A. K. and McDonagh, A. F.: Phototherapy for neonatal hyperbilirubinemia: efficacy, mechanism and toxicity. Avd. Pediat., 27: 341 (1980).

3. Cremer, R. J., Perryman, P. W., and Richards, D. K.: Influence of light on the hyperbilirubinemia of infants. Lancet, $i: 1094$ (1958).

4. Ostrow, J. D.: Photocatabolism of labeled bilirubin in the congenitally jaundiced Gunn rat. J. Clin. Invest., 50: 707 (1971)

5. Lund, H. T. and Jacobsen, J.: Influence of phototherapy on the biliary bilirubin excretion pattern in newborn infants with hyperilirubinemia. J. Pediatr. 85 : 262 (1974).

6. Ostrow, J. D., Berry, C. S., and Zarembo, J. E.: Studies on the mechanism of phototherapy in the congenitally jaundiced rat. In: Odell G. B., Schaffer, R., Simoupoulis, A., (Eds.). Phototherapy in the Newborn: an overview. pp. 74-92 (National Academy of Sciences, Washington D.C., 1974).

7. McDonagh, A. F., and Ramonas, L. M.: Jaundice phototherapy: microflow-cell photometry reveals rapid biliary response of Gunn rats to light. Science 201: 829 (1978).

8. Stoll, M. S., Zenone, E. A., Ostrow, J. D., and Zarembo, J. E.: Preparation and properties of bilirubin photoisomers. Biochem. J., 183: 139 (1979).

9. Onishi, S., Kawade, N., Itoh, S., Isobe, K., and Sugiyama, S.: High-pressure liquid chromatographic analysis of anaerobic photo-products of bilirubin-IXa in vitro and its comparison with photo-products in vivo. Biochem. J., 190: 527 (1980).

10. Erlinger, S. D., Dhumeaux, J. F., and Benhamou, J. P.: Hepatic handling of unconjugated dyes in the Dubin-Johnson syndrome. Gastroenterology, 64: 106 (1973).

11. Lester, R. and Schmid, R.: Intestinal absorption of bile pigments. I. The enterohepatic circulation of bilirubin in the rat. J. Clin. Invest., 42: 736 (1963).

12. Poland, R. L. and Odell, G. B.: Physiologic jaundice: the enterohepatic circulation of bilirubin. N. Engl. J. Med. 284: 1 (1971).

13. Windorfer, A. Jr., Kunzer, W., Bolze, H., Ascher, K., Wilcken, F., and Hoehne, K.: Studies on the effect of orally administered agar on the serum bilirubin level of premature infants and mature newborns. Acta Paediatr. Scand., 64: 699 (1975).

14. Maurer, H. M., Shumway, C. N., Draper, D. A., and Hossaini, A. A.: Controlled trial comparing agar, intermittent phototherapy and continuous phototherapy for reducing neonatal hyperbilirubinemia. J. Pediatr., 82: 73 (1973).

15. Schellong, G., Quakermack, K., and Fuhrmans, B.: The influence of agar feeding on serum bilirubin in physiologic jaundice of the newborn infant. Z. Geburtshilfe Perinatol., 178: 34 (1974).

16. Romagnoli, C., Polidori, M., Foschini, L., Cataldi, P., de Turics, P., Tortorolo, G., and Mastrangelo R.: Agar in the management of hyperbilirubinemia in the premature baby. Arch. Dis. Child. 50: 202 (1975).

17. Meloni, T., Costa, S., Corti, R., and Cutillo, S.: Agar in control of hyperbilirubinemia of full-term newborn infants with erythrocyte G-6-PD deficiency. Biol. Neonate, 34: 295 (1978).

18. Ebbesen, F., Miller, J. Agar ingestion combined with phototherapy in jaundiced newborn infants. Biol. Neonate 31: 7-9 (1977)

19. Poland, R. L., and Odell, G. B.: The binding of bilirubin to agar. Proc. Soc. Exp. Biol. Med., 146: 1114 (1974)

20. Odell, G. B., Bolen, J. L., Poland, R. L., Seungdamrong S., and Cukier J. O.: Protection from bilirubin nephropathy in jaundiced Gunn rats. Gastroenter- 
ology, 66: 1218 (1974)

21. Odell, G. B.: Neonatal hyperbilirubinemia. pp. $44-47$ (Grune and Stratton, New York, 1980).

22. Cukier, J. L., Maglalang, A. C., and Odell, G. B.: Increased osmotic fragility of erythrocytes in chronically jaundiced rats after phototherapy. Acta Paediatr. Scand. 68: 903 (1979).

23. Malloy, H. T., Evelyn, K. A.: The determination of bilirubin with the photoelectric colorimeter. J. Biol. Chem. 119: 481 (1937).

24. Schwarz, M. P., von Bergman, K., and Paumgartner, G.: A simple method for the estimation of bile acids in serum. Clin. Chim. Acta, 50: 197 (1974).

25. Boyer, J., Baron, D. N., and Talalay, P.: Purification and properties of a $3 \alpha-$ hydroxysteroid dehydrogenase from Pseudomonas testosteroni. Biochemistry, 4: 1825 (1965)

26. Brownlee, K. A.: Industrial experimentation. 4th edition (Chemical Publishing Co., New York, 1953)

27. Dixon, W. J., Massey, F. J., Jr.: Introduction to statistical analysis. 3rd edition (McGraw-Hill Co., New York, 1969).

28. Arrowsmith, W. A., Payne, R. B., and Littlewood, J. M.: Comparison of treat- ments for congenital non-obstructive non-haemolytic hyperbilirubinemia. Arch. Dis. Child. 50: 197 (1975).

29. McDonagh, A. F., Palma, L. A., and Lightner, D. A.: Blue light and bilirubin excretion. Science, 208: 145 (1980).

30. John, E.: Complications of phototherapy in neonatal hyperbilirubinemia. Aust. Paediatr. J., 11: 53 (1975).

31. Rubaltelli, F. F., and Largajolli, G.: Effect of light exposure on gut transit time in jaundiced newborns. Acta Paediatr. Scand., 62: 146 (1973).

32. Whitington, P. F., Olsen, W. A., and Odell, G. B.: The effect of bilirubin on the function of hamster small intestine. Pediatr. Res., 15: 1009 (1981).

33. Li, B., Whitington, P. F., and Odell, G. B.: Reversal of bilirubin-induced small bowel secretion by agar. Pediatr. Res., 13: 403A (1979).

34. Requests for reprints should be addressed to: Dr. G. B. Odell, Department of Pediatrics, University of Wisconsin Clinical Science Center, 600 Highland Avenue, Madison, WI 53792 USA.

35. These studies have been supported by USPHS Grant AMDD \#21668.

36. Received for publication April 24, 1981.

37. Accepted for publication March 1, 1983

\title{
A Mechanism for Liver Cell Injury in Viral Hepatitis: Effects of Hepatitis B Virus on Neutrophil Function in Vitro and in Children with Chronic Active Hepatitis
}

\author{
ALBERTO VIERUCCI, MAURIZIO DE MARTINO, ELISA GRAZIANI, MARIA E. ROSSI, W. THOMAS \\ LONDON, AND BARUCH S. BLUMBERG ${ }^{(63)}$
}

\author{
Division of Immunology, Institute of Pediatrics, University of Florence, Florence, Italy, and Institute for Cancer
}

Research, Fox Chase Cancer Center, Philadelphia, Pennsylvania USA

\begin{abstract}
Summary
Neutrophil function was studied by nitroblue tetrazolium (NBT) reduction, superoxide anion $\left(\mathrm{O}_{2}{ }^{\bar{\sigma}}\right)$ production, chemotaxis, and bactericidal activity in 9 children with HBsAg-positive chronic active hepatitis (CAH). NBT reduction and $\mathrm{O}_{2}^{-}$production were higher in resting neutrophils from the children with $\mathrm{CAH}$ than from the controls, but the production of $\mathrm{O}_{2}{ }^{-}$was depressed after stimulation with zymosan. No defect was observed in both random and direct locomotion, whereas a significant decrease was present in bactericidal activity. To evaluate the role of virus components, purified preparations of $\mathrm{HBsAg}$ and $\mathrm{HBcAg}$ were added to neutrophils from normal children. The incubation with such products induced in vitro the same modifications that were observed in the neutrophils from children with CAH. In addition, defects in phagocytosis and killing of Candida albicans as well as in chemotaxis were demonstrated. The production of $\mathrm{O}_{2}{ }^{-}$was reduced in the neutrophils, stimulated by zymosan and previously opsonized with HBsAg-positive serum.
\end{abstract}

\section{Abbreviations}

CAH, chronic active hepatitis

HBcAg, hepatitis B core antigen

HBsAg, hepatitis B surface antigen

HBV, hepatitis B virus

KRP, Krebs Ringer Phosphate
NBT, nitroblue tetrazolium

PMA, phorbolmyristate acetate

PMNs, polymorphonuclear neutrophils

SGOT, serum aspartate aminotransferase

SGPT, serum alanine aminotransferase

The mechanism by which HBV causes liver damage is unclear (35). Because individuals with persistent HBV infection and large numbers of virus particles in their blood frequently have little or no liver damage but patients with transient infections often have low titers of circulating HBV particles and severe liver damage, most investigators have concluded that viral replication, itself, is not the cause of liver cell death. Because patients with impaired immune functions (lepromatous leprosy, end stage renal disease, leukemia, etc.) are more likely to develop persistent infections than persons without impaired immune responses, several investigators have proposed that it is the immune response to HBV that is responsible for the damage to hepatocytes $(8,40)$. Recently, Dienstag et al. (14) and Levy and Chisari (35) reviewed various hypotheses that have been proposed to explain how lymphocytes or antibodies might be involved in the liver damage of hepatitis. They concluded that there was insufficient evidence to support any particular cellular or humoral mechanism.

An alternative possibility is that phagocyte cells, i.e., PMNs and macrophages, may be involved in at least type B hepatitis. Alter- 